\title{
Control Chaos of a Unidirectionally Traffic Coupled Map Lattice Model
}

\author{
Yaling Fang \\ College of Electrical and Power Engineering, Taiyuan University of Technology, Taiyuan, China \\ Email: lianhao1227@163.com
}

How to cite this paper: Fang, Y.L. (2021) Control Chaos of a Unidirectionally Traffic Coupled Map Lattice Model. Journal of Applied Mathematics and Physics, 9, 2527-2533. https://doi.org/10.4236/jamp.2021.910162

Received: September 4, 2021

Accepted: October 24, 2021

Published: October 27, 2021

Copyright $\odot 2021$ by author(s) and Scientific Research Publishing Inc. This work is licensed under the Creative Commons Attribution International License (CC BY 4.0).

http://creativecommons.org/licenses/by/4.0/

\begin{abstract}
Spatiotemporal chaos is studied by using the unidirectional traffic coupled lattice model with hyperbolic tangent local map. The coupled map lattice (CML) model can simulate the complex traffic flow phenomenon which is similar to the traditional traffic flow model. The nonlinear feedback method is used to study the control of the chaotic system of the unidirectionally traffic coupled map lattice model. The stability of spatiotemporal chaos in the coupled map lattice is realized. The results of numerical simulation show that there is a relationship between control results and control parameters when controlling spatiotemporal chaos to a uniform stable state in a certain phase space compression parameter region.
\end{abstract}

\section{Keywords}

Traffic Flow Model, Coupled Map Lattice Model, Spatiotemporal Chaos, Feedback Control

\section{Introduction}

The problem of heavy traffic has aroused a wide concern [1] [2]. There is always no obvious reason for traffic jams that every driver experiences. Traffic congestion is the most typical complicated problem in traffic flow. Traffic flow is a many-body system with strong interaction. The current research finds that traffic flow has the following characteristics, such as phase transition, nonlinear wave and chaos [3] [4]. The main methods of traffic flow modeling are as follows: gas kinetic models, hydrodynamic models, cellular automaton models and car following models [5] [6] [7].

Comparing with other chaotic systems, spatiotemporal chaos is of higher dimension, and has more complex dynamic properties. Coupled map lattice is a kind of dynamical system array with discrete time, which is a simple model of 
spatiotemporal complexity [8] [9]. CMLs can not only render extremely rich spatiotemporal behavior of chaotic dynamics, but also easy to model dynamics in fluid phenomena such as turbulence [10]. CMLs are an understanding of the basic characteristics of flow system, CMLs describes basic flow systems, which is more convenient for numerical or analytical processing. There are many chaos control method research about time chaos which developed faster and faster, but for spatiotemporal chaos research is relatively lacking. And the spatiotemporal chaos is more complicated than time chaos. The OGY method [11] for controlling spatiotemporal chaos of logistic maps is proposed. Pinning is another way to control spatiotemporal time chaos in CMLs [12]. The OGY method and its improvement methods all perform time perturbation feedback on the parameters of the system and achieve the purpose of controlling some unstable periodic orbits. This requires finding a suitable system parameter for perturbation adjustment. But in fact not all nonlinear systems can find adjustable parameters. Many systems in practice are difficult to find their suitable tunable parameters. Parekh and Sinha extended the spatiotemporal chaos control of the coupled map lattice to anti-control, and the coupled map lattice changes from a non-chaotic state to a chaotic state, or weakly in the coupled map lattice [13]. Takashi Nagatani [14] uses a unidirectionally coupled hyperbolic tangent map lattice to prove that the dynamic sensitivity to boundary disturbance makes different traffic states have certain spatiotemporal characteristics. But the control of the coupled lattice model is not considered in previous studies.

The aim of the present work is to prove that the chaotic features of the traffic unidirectionally coupled map lattice model are studied and its spacio-temporal characteristic is analyzed, which reflects the characteristics of traffic flow. A discrete-time controller for controlling spatiotemporal chaos of one way CMLs is introduced.

\section{The Unidirectionally Traffic Coupled Map Lattice Model}

We model a unidirectionally one-way CML which has a large coupling coefficient greater than one because CML demonstrates the confusion caused by its structure and closely relates to traffic flow model. A one-dimensional traffic flow is described by a one-way CML. It is assumed that $V\left(\Delta x_{j}(t)\right)$ is the optimal velocity, which $x_{j}(t), \Delta x_{j}(t)$ and $h_{c}$ represent the position of vehicle $j$, the headway and the safety distance respectively.

The optimal velocity function is adopt as follows [6]

$$
V\left(\Delta x_{j}(t)\right)=\frac{v_{\max }}{2}\left[\tanh \left(\Delta x_{j}(t)-h_{c}\right)+\tanh \left(h_{c}\right)\right]
$$

where $v_{\max }$ is the maximum speed.

The legal velocity $V\left(\Delta x_{j}(t)\right)$ of vehicle number $n$ depends on the following distance of the preceding vehicle number $n+1$. When the gap is smaller, the speed must be reduced to a small enough to prevent it from hitting the vehicle in front. On the other hand, when the headway is longer, the vehicle can travel at a 
higher speed, but not exceeding the maximum speed. The situation is more objective, quantitative and practical.

We suppose that the headway $\Delta x_{j}(t+1)$ at time $t+1$ is determined by

$$
\Delta x_{j}(t+1)-h_{c}=V\left(\Delta x_{j}(t)\right)-V\left(h_{c}\right)
$$

The variables and functions are defined as

$$
u_{j}(t)=\Delta x_{j}(t)-h_{c} \text { and } f(u)=V\left(u+h_{c}\right)-V\left(h_{c}\right)
$$

The dynamical system is approximately described by the hyperbolic tangent map

$$
f(u)=\frac{v_{\max }}{2} \tanh (u)
$$

The local mapping $f$ has two stable equilibria.

The variable $\varepsilon$ is introduced to describe that vehicle $j$ changes the headway $\Delta x_{j}(t)$ When the headway $\Delta x_{j}(t+1)$ of vehicle ahead $j+1$ changes. The vehicle $j$ proceeds ahead by the amount $\varepsilon\left[V\left(\Delta x_{j+1}(t)\right)-V\left(\Delta x_{j}(t)\right)\right]$.

The distance is added to the right side of Equation (2)

$$
u_{j}(t+1)=(1-\varepsilon) f\left(u_{j}(t)\right)+\varepsilon f\left(u_{j+1}(t)\right)
$$

Thus, the traffic flow model in CML form is established.

In a general, the model can be written as follows when parameter $\alpha=1$

$$
u_{j}(t+1)=(1-\varepsilon) f\left(u_{j}(t)\right)+\varepsilon\left[(1-\alpha) f\left(u_{j-1}(t)\right)+\alpha f\left(u_{j+1}(t)\right)\right]
$$

Note that $u_{j}(t)$ is governed only by a single delayed map (5), where $\varepsilon$ is the weight of the current state $f\left(u_{j}(t)\right)$ and the past state $f\left(u_{j+1}(t)\right)$.

\section{Controlling Spatiotemporal Chaos to the Homogeneous State}

The unidirectionally traffic coupled map lattice model (6) is fully developed for turbulent flow patterns when $v_{\max }=1, \varepsilon=0.5$. Consider generality, $\alpha \in[0,1]$ is a parameter controlling the symmetry of coupling in (6). For this model, the following nonlinear local feedback control method is adopted, i.e., the following control strategy is adopted for the system (6)

$$
\begin{aligned}
u_{j}(t+1)= & (1-\varepsilon) f\left(u_{j}(t)\right)+\varepsilon\left[(1-\alpha) f\left(u_{j-1}(t)\right)+\alpha f\left(u_{j+1}(t)\right)\right] \\
& -k\left(f\left(u_{j}(t)\right)-u_{j}(t)\right)
\end{aligned}
$$

Which $k$ is feedback coefficient.

Obviously, the control term does not change the fixed points of the original system.

\subsection{The Stability Analysis of CML}

Let us consider the one-dimensional maps (7). Because the boundary conditions affect the Jacobin matrix, we choose a fixed boundary condition in this section to simplify the theoretical analysis. Fixed boundary condition means that $u_{n}^{0}$ and 
$u_{n}^{N+1}$ are fixed at $u_{n}^{0}=u_{n}^{N+1}=u_{f}$, where $u_{f}$ is the fixed point of the local map, i.e., $u_{f}=f\left(u_{f}\right)$. The steady state of $(7)$ is

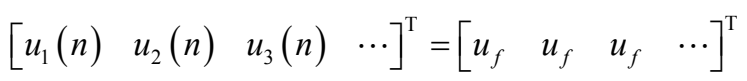

The dynamics of ith lattice site is focused. It is assumed that the dynamics of the sites (i.e., $1,2, \cdots,(i-2)$ th lattice sites) have already converged to the fixed point (i.e., $u_{m}(n)=u_{f}$ for $m=1,2, \cdots,(i-2)$ ). Assuming that $u_{i-1}(n)$ and $u_{i}(n)$ are in the neighborhood of the fixed point $u_{f}$, the dynamics of the $i$ th lattice site can be given as

$$
y_{i}(n+1)=(1-\varepsilon-k) \Lambda y_{i}(n)+\varepsilon \Lambda y_{i-1}(n)
$$

where

$$
y_{i}(n):=u_{i}(n)-u_{f}, \quad y_{i-1}(n):=u_{i-1}(n)-u_{f}, \quad \Lambda:=\left.\frac{\partial f(u)}{\partial u}\right|_{u=u_{f}}
$$

The $\mathrm{Z}$ transforms [15] of $y_{i}(n+1), y_{i}(n)$ and $y_{i-1}(n)$ are as follows

$$
Z\left[y_{i}(n+1)\right]=z Y_{i}(z), \quad Z\left[y_{i}(n)\right]=z Y_{i}(z), Z\left[y_{i-1}(n)\right]=Y_{i-1}(z)
$$

Taking the $Z$ transforms of both sides of Equation (8)

$$
z Y_{i}(z)=(1-\varepsilon-k) \Lambda Y_{i}(z)+\varepsilon \Lambda Y_{i-1}(z)
$$

The relation of $Y_{i}(z)$ and $Y_{i-1}(z)$ is described as

$$
Y_{i}(z)=G(z) Y_{i-1}(z)
$$

Where the transfer function $G(z)$ is

$$
G(z)=\frac{\varepsilon \Lambda}{z-(1-\varepsilon-k) \Lambda}
$$

When the $(i-1)$ th lattice site is fixed at $u_{i-1}(n)=u_{f}$, the $i$ th lattice site state $u_{i}(n)$ converges on $u_{f}$ only when the pole of $G(z)$ is in the unit circle on complex plane.

$$
|(1-\varepsilon-k) \Lambda|<1
$$

From Equation (6), we have

$$
\frac{\partial u_{n+1}^{i}}{\partial u_{n}^{i}}=(1-\varepsilon) \Lambda-k(\Lambda-1)
$$

Denote $M\left(U_{f}\right)$ [15] as the Jacobian matrix of Equation (7) at the homogeneous state

$$
M\left(U_{f}\right)=\left(\begin{array}{cccccc}
a & b & 0 & \cdots & 0 & 0 \\
c & a & b & \cdots & 0 & 0 \\
0 & c & a & \cdots & 0 & 0 \\
\vdots & \vdots & \vdots & \ddots & \vdots & \vdots \\
0 & 0 & 0 & \cdots & a & b \\
0 & 0 & 0 & \cdots & c & a
\end{array}\right)
$$

where $a=(1-\varepsilon) \Lambda-k(\Lambda-1), \quad b=\varepsilon(1-\alpha) \Lambda, \quad c=\varepsilon \alpha \Lambda$. 
The eigenvalues of Jacobian matrix $M\left(U_{f}\right)$ is

$$
\lambda_{i}=a+2 b \sqrt{c / b} \cos \left(\frac{s \pi}{N+1}\right), \quad s=1, \cdots, N
$$

A stable homogeneous state requires $\left|\lambda_{i}\right|<1$ for all $i$.

When $N$ is large,

$$
\begin{aligned}
& \lambda_{\text {min }}=\min \left(\lambda_{1}, \lambda_{1}, \cdots, \lambda_{N}\right) \approx a-2 b \sqrt{c / b} \\
& \lambda_{\text {max }}=\max \left(\lambda_{1}, \lambda_{1}, \cdots, \lambda_{N}\right) \approx a+2 b \sqrt{c / b}
\end{aligned}
$$

For Equation (12), Equation (13) and Equation (14) lead to

$$
\frac{-1-(1-\varepsilon) \Lambda+2 \varepsilon(1-\alpha) \Lambda \sqrt{\alpha /(1-\alpha)}}{1-\Lambda}<k<\frac{1-(1-\varepsilon) \Lambda-2 \varepsilon(1-\alpha) \Lambda \sqrt{\alpha /(1-\alpha)}}{1-\Lambda}
$$

The controlling parameter $k$ is determined according to the above inequality (15) when the values of $\alpha$ and $\varepsilon$ are known. Inequality (15) gives a method for successfully controlling spatiotemporal chaos by selecting appropriate control parameters. Inequality (15) is a sufficient condition for a stable homogeneous state.

\subsection{Realization of Spatiotemporal Chaos Control}

When the control is turned off, the spatiotemporal chaotic behavior in CML (6) becomes the homogeneous state [16] [17]. The control method works well for an asymmetric coupled CML with $\alpha=0.1$. Controlling for other situations is also successful. If $v_{\max }=1, \varepsilon=0.5$ and $\alpha=0.1$, inequality (11) and (15) means $0.32<k<0.97$. If $k$ is chosen in $(0.32,0.97)$, the homogeneous state becomes stable.

Figure 1(a) shows the spatial and temporal behavior development diagram of the system without control. As can be seen from the figure, the system is obviously chaotic in time and space. The theoretical results are in good agreement

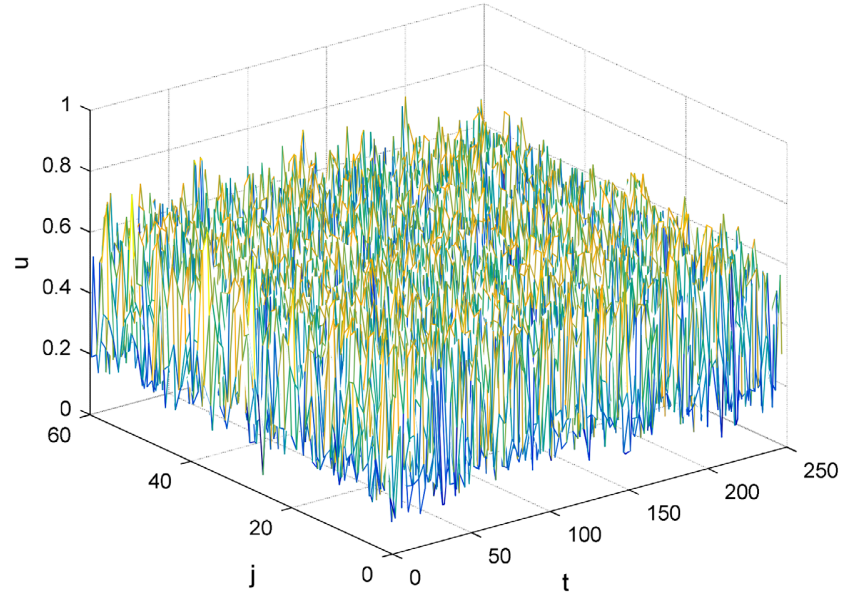

(a)

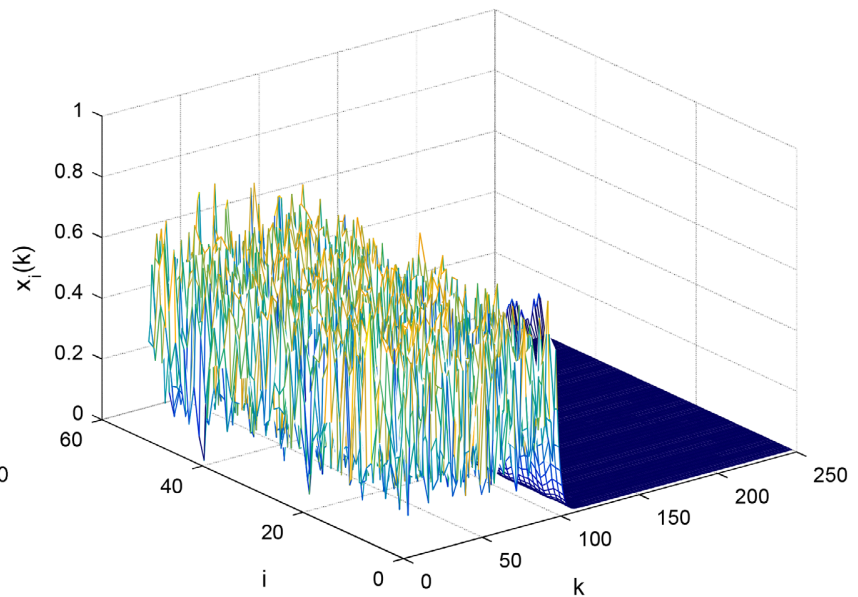

(b)

Figure 1. Spatial and temporal behavior (a) without control (b) under control ( $k=0.4$ ). 
with the numerical simulation results of asymmetric coupling CML with $\alpha=0.1$. The homogeneity is that different spatial lattices have the same state at the same time, and the stationary state is that the same spatial lattices have the same state at different times. In Figure 1(b), the horizontal coordinates of the spatial state graph are the spatial image grid, and the vertical coordinates are the state variables of each image grid at the last moment. In the numerical simulation of this paper, all initial values are random uniformly distributed on the interval $[-1,1]$. Figure 1(b) shows the simulation result, and controller is applied after 100 steps of free evolution.

\section{Conclusion}

A new traffic flow model is modelled according to the general characteristics of traffic flow. This model can simulate the spatiotemporal chaos in traffic flow. The stable control of spatiotemporal chaos in coupled map lattices is realized by nonlinear state feedback method. The numerical simulation results given above fully demonstrate the effectiveness of this control method. This control method is very flexible. Different combinations of feedback methods, control methods and control intensity can achieve different control goals, which is also very necessary in practical application. Through spatial coupling, other uncontrolled spatial points are also brought to the target state, and the control of space-time chaos is finally realized.

\section{Acknowledgements}

The work is supported by Shanxi Province Science Foundation for Youths (Grant No.201801D221181).

\section{Conflicts of Interest}

The author declares no conflicts of interest regarding the publication of this paper.

\section{References}

[1] Roncoli, C., Papageorgiou, M. and Papamichail, I. (2015) Traffic Flow Optimisation in Presence of Vehicle Automation and Communication Systems-Part I: A First-Order Multi-Lane Model for Motorway Traffic. Transportation Research Part C: Emerging Technologies, 57, 241-259. https://doi.org/10.1016/j.trc.2015.06.014

[2] Orosz, G., Wilson, R.E. and Stépán, G. (2010) Traffic Jams: Dynamics and Control. Philosophical Transactions of the Royal Society A, 368, 4455-4479. https://doi.org/10.1098/rsta.2010.0205

[3] Redhu, P. and Gupta, A.K. (2015) Jamming Transitions and the Effect of Interruption Probability in a Lattice Traffic Flow Model with Passing. Physica A: Statistical Mechanics and Its Applications, 421, 249-260. https://doi.org/10.1016/j.physa.2014.11.037

[4] Fang, Y.L., Shi, Z.K. and Cao, J.L. (2015) Congestion Phenomenon Analysis and Delayed-Feedback Control in a Modified Coupled Map Traffic Flow Model Con- 
taining the Velocity Difference. Communications in Nonlinear Science and $\mathrm{Nu}$ merical Simulation, 23, 175-184. https://doi.org/10.1016/j.cnsns.2014.11.007

[5] Fang, Y. and Shi, Z. (2015) Chaos Analysis and Delayed-Feedback Control in a Discrete Dynamic Coupled Map Traffic Model. Physica A: Statistical Mechanics and Its Applications, 422, 40-46. https://doi.org/10.1016/j.physa.2014.11.038

[6] Bando, M., Hasebe, K., Nakayama, A., et al. (1995) Dynamical Model of Traffic Congestion and Numerical Simulation. Physical Review E, 51, 1035. https://doi.org/10.1103/PhysRevE.51.1035

[7] Tian, J., Li, G., Treiber, M., et al. (2016) Cellular Automaton Model Simulating Spatiotemporal Patterns, Phase Transitions and Concave Growth Pattern of Oscillations in Traffic Flow. Transportation Research Part B: Methodological, 93, 560-575. https://doi.org/10.1016/j.trb.2016.08.008

[8] Redhu, P. and Gupta, A.K. (2015) Delayed-Feedback Control in a Lattice Hydrodynamic Model. Communications in Nonlinear Science and Numerical Simulation, 27, 263-270. https://doi.org/10.1016/j.cnsns.2015.03.015

[9] Biswas, S. and Das, A. (2016) Patterns, Bifurcations, Multistability and Hysteresis in an Inhomogeneous Coupled Map Lattice. International Journal of Bifurcation and Chaos, 26, Article ID: 1630008. https://doi.org/10.1142/S0218127416300081

[10] Paul, S. and Mitra, A. (2017) Generating Efficient Techniques for Information Extraction and Processing Using Cellular Automata. In: Bio-Inspired Computing for Information Retrieval Applications, IGI Global, Hershey, 204-228.

https://doi.org/10.4018/978-1-5225-2375-8.ch008

[11] de Paula, A.S. and Savi, M.A. (2009) A Multi Parameter Chaos Control Method Based on OGY Approach. Chaos, Solitons \& Fractals, 40, 1376-1390. https://doi.org/10.1016/j.chaos.2007.09.056

[12] Grigoriev, R.O., Cross, M.C. and Schuster, H.G. (1997) Pinning Control of Spatiotemporal Chaos. Physical Review Letters, 79, 2795.

https://doi.org/10.1103/PhysRevLett.79.2795

[13] Parekh, N., Parthasarathy, S. and Sinha, S. (1998) Global and Local Control of Spatiotemporal Chaos in Coupled Map Lattices. Physical Review Letters, 81, 1401. https://doi.org/10.1103/PhysRevLett.81.1401

[14] Nagatani, T. (2001) Phase Diagrams in Unidirectionally Coupled Map Lattice for Open Traffic Flow. Physica A: Statistical Mechanics and Its Applications, 289, 267-277. https://doi.org/10.1016/S0378-4371(00)00497-0

[15] Smith, G.D. (1985) Numerical Solution of Partial Differential Equations: Finite Difference Methods. Oxford University Press, Oxford.

[16] Zhu, K.E. and Chen, T. (2001) Controlling Spatiotemporal Chaos in Coupled Map Lattices. Physical Review E, Statistical, Nonlinear, and Soft Matter Physics, 63, Article ID: 067201. https://doi.org/10.1103/PhysRevE.63.067201

[17] Kek, S.L., Sim, S.Y. and Chen, C.Y. (2019) An Expanded Optimal Control Policy for a Coupled Tanks System with Random Disturbance. Advances in Pure Mathematics, 9, 317-329. https://doi.org/10.4236/apm.2019.94014 